\title{
FACTORS RELATED TO DEFAULTERS AND TREATMENT FAILURE OF TUBERCULOSIS PATIENTS IN THE DOTS PROGRAM IN THE SUNSARI DISTRICT OF EASTERN NEPAL
}

\author{
Lamsal DK, ${ }^{1 \cdot}$ Lewis OD, ${ }^{2}$ Smith $S,{ }^{3}$ Jha ${ }^{4}$ \\ ${ }^{1}$ Lecturer, Kathmandu Medical College, Kathmandu \\ ${ }^{2}$ Professor, Department of Family Medicine, B. P. Koirala Institute of Health Sciences, Dharan, Nepal \\ ${ }^{3}$ Associate Professor, Department of Family Medicine, B. P. Koirala Institute of Health Sciences, Dharan, Nepal \\ ${ }^{4}$ Head of Department of Community Medicine, B. P. Koirala Institute of Health Sciences, Dharan, Nepal
}

\begin{abstract}
Objectives : The aim of this study was to investigate reasons underlying failure to complete DOTS treatment in TB patients in Dharan and the Sunsari district of eastern Nepal.

Methods : A descriptive, qualitative study using semi structured questionnaires after identifying cases from register data. Results were then compared with the results of informal recorded discussions, direct observation, and focus group discussions. All defaulter and treatment failure TB patients in the DOTS program in Sunsari district from July 2002 to July 2003 were included in the study.

Results : All of the defaulter patients in this study were struggling financially, and said that they had trouble leaving work to go to the DOTS centre, as well as difficulty with distance and transport. Other issues such as alcohol, stigma, poverty, inadequate education and the use of private clinics and traditional healers were discussed.

Conclusion : Almost half the Nepalese population is infected with TB. Despite meeting the WHO goals for default and treatment failure it is important to aggressively address those aspects that can be changed so the country can continue to reduce the burden of TB on its community. Recommendations are made from the results of this study that may help improve adherence to DOTS programmes.
\end{abstract}

Keywords : tuberculosis, Nepal, DOTS, defaulter, compliance

\section{INTRODUCTION}

Tuberculosis (TB) is major public health problem worldwide. The number of cases of TB is increasing by $2.4 \%$ per year with nearly 8.9 million new cases in 2004. ${ }^{1}$

\section{Correspondence to}

Dr. Dinesh Kumar Lamsal

Lecturer, Kathmandu Medical College, Kathmandu lamsald@hotmail.com

lamsaldb@yahoo.com/niljha@hotmail.com
In Nepal it is estimated that $45 \%$ of the total population is infected with TB. 44,000 people develop active TB and 8-11,000 people die each year from this disease. ${ }^{1}$ Detection rates are still significantly low - at least in part because of reliance on sputum smears that can only ever detect a proportion of cases. ${ }^{1}$ Pulmonary TB accounts for only $36-48 \%$ of all people with TB and smear microscopy only detects $45-60 \%$ of these. ${ }^{1}$

DOTS (Directly Observed Treatment, Short course) was implemented by the World Health Organisation (WHO) in South Asia in 1993-94 and in Nepal in 1996. ${ }^{1}$ Its five elements are political commitment; case 
detection by sputum smear microscopy; a system to ensure regular drug supplies; a standard recording and reporting system, including the assessment of treatment outcomes and standard short-course chemotherapy administered under standardised case-management conditions. ${ }^{1}$ It is now integrated into the general health services and covers $89 \%$ of the Nepalese population. In Eastern Nepal it is run in 46 treatment centres and 253 sub centres. ${ }^{2}$

The goal of DOTS is to reduce transmission in the community by aiming for cure of at least $85 \%$, and detection of at least $70 \%$ of new smear positive cases. ${ }^{2}$ The purpose of directly observed therapy is to increase the probability of treatment completion. - and to thereby avoid treatment failure, persistent infectivity, drug resistance and relapse. ${ }^{1}$ When based in primary health centres DOTS has been shown to have equivalent outcomes and to be more cost effective than treatment regimes based on in-patient supervision or large hospital centres. ${ }^{1}$

About $50 \%$ of those infected with TB will die within five years without intervention as compared to only 2-3\% of those receiving DOTS treatment. According to year 2000 figures from the Nepalese National Tuberculosis Centre, the treatment success rate for the DOTS program is about $89 \%$, the defaulter rate $6 \%$ and the rate of treatment failure $1 \%{ }^{2}$

Multi Drug Resistant(MDR)-TB is defined as resistance to both the first-line TB drugs, Isoniazid and Rifampicin. Since the early 1990's MDR-TB has been on the rise and now threatens TB control programs in many parts of the world. It can be primary, in a patient who has not been treated before, reflecting overall poor TB control in the community, or acquired, reflecting either poor adherence or an inadequately conducted individual program. MDR-TB makes management even more difficult because of the need for longer courses of less potent, more toxic and more expensive second line drugs. ${ }^{4}$ Proposed explanations for the rise in MDR-TB include HIV, physician mismanagement, substance abuse and failure to complete therapy due to incomplete or inadequate therapy. ${ }^{1}$ The prevention and control of MDR-TB is one of the aims of the 2006 WHO "Stop TB" strategy. ${ }^{1}$

Poverty and tuberculosis are intimately connected. ${ }^{5}$ The poor have higher contact rates due to crowded homes, more active infection due to sub-optimal nutrition and working conditions, and they frequently have less access to diagnostic and treatment facilities. ${ }^{5}$ They may have less flexibility regarding work and clinic attendance and less ability to pay for medications and transport. A study conducted in an urban area of India in 2000-01 found one quarter of their non-compliant patient were the only economically active person in the family and had no spare time to visit DOTS centres. ${ }^{6}$

The TB control program in Vietnam found that $20 \%$ of their defaulters lacked knowledge about the disease and its treatment. ${ }^{7}$ A similar study in Nepal found that adherent patients seemed to be better informed about their disease. ${ }^{8}$ Illiteracy and difficulty in understanding the information provided by the health worker have been linked with non-compliance. ${ }^{6}$

One way of dealing with this lack of understanding is to increase the level of knowledge in the whole community. A study on the level of knowledge about TB in a rural community in India found that $73.7 \%$ knew that cough with sputum was the most common symptom of TB, $95.3 \%$ were aware that TB could spread to others but only $48 \%$ knew it was caused by an infectious agent. TB patients share the community's cultural beliefs about causes of TB and its spread. A common misconception was that separate utensils and food were necessary for TB patients. ${ }^{9}$ Some may choose to seek help from traditional healers. The stigma of the disease is likely to be influenced by many of these beliefs and decisions. ${ }^{10}$

Alcoholic patients are more likely to be irregular in their compliance with treatment. A study in India found $28 \%$ of defaulters were alcoholic. Distance from the health centre and inconvenient office hours have also been found to be important in treatment compliance 
with DOTS therapy. ${ }^{11}$ Patients often discontinue TB medication because of such side effects as weakness, GI upset, allergy, jaundice and passing of red urine. ${ }^{?}$

Ineffective or inappropriate prescriptions of TB drugs are very common outside the DOTS program. In India it was found that private prescriptions commonly contained errors and that multiple brands and combinations of drugs led to confusion for patients. In one study $81 \%$ of pharmacies had been dispensing private anti-tuberculosis prescriptions and 95\% were not aware of the existence of the DOTS program. In the same study $55-62 \%$ of individuals with chest symptoms initially approached private medical practitioners. ${ }^{12}$ The aim of this study was to investigate reasons underlying failure to complete DOTS treatment in TB patients in Dharan and the Sunsari district of eastern Nepal.

\section{MATERIALS AND METHODS}

The study was undertaken in the teaching district of B.P.Koirala Institute of Health Sciences (BPKIHS), Dharan.

Study design: A Descriptive, Qualitative study using semi structured questionnaires after identifying cases from register data. Results were then compared with the results of informal recorded discussions, direct observation, and focus group discussions.

All defaulter and treatment failure TB patients in the DOTS program in Sunsari district from July 2002 to July 2003 were included in the study. Of the 1040 registered TB cases in Sunsari district there were 35 cases eligible for inclusion as well as 23 relatives and DOTS health staff. TB cases continuing with or completing the DOTS program during the study period were excluded from the sample.

Treatment failure was defined as a patient who is still sputum smear positive five months or more after the commencement of treatment. A defaulter was defined as a patient whose treatment was interrupted for two consecutive months or more. ${ }^{2}$

Information was obtained from unstructured interviews with respondents at home (patients' relatives, patients themselves); in-depth interviews using a questionnaire with defaulters, treatment failure patients, key informants, and DOTS workers; focus group discussions with patients and relatives; and observation in DOTS Centres and homes by the researcher.

DOTS centres case finding and treatment records were analyzed in collaboration with the health workers. The distance from DOTS centres and the DOTS worker's opinion about the reason for those who defaulted or failed treatment were recorded. All individual defaulter and treatment failure cases were visited and interviewed.

The questionnaire in English was designed after consultation with DOTS workers, then translated into Nepali by the researcher and pre-tested on patients at a DOTS clinic. The questions were re-translated back to English, by a translator to maintain consistency in the meaning.

Focus group discussions were held with four groups: defaulters, relatives of defaulters, those living in urban areas close to a DOTS centre and those living in more distant rural areas.

\section{RESULTS}

Of the 1040 registered cases of TB in the Sunsari district (population 588,212), there were 27 defaulters, eight treatment failures and two patients who died during the study. Of the 344 TB cases in Dharan (population 103,012), there were 12 defaulters and four treatment failures. Four PHC, three $\mathrm{HC}$, thirty three DOTS centres and sub-centres were visited.

Results of focus group discussions : Participants from all groups were aware that TB is a communicable 
and curable disease, they wear also aware of the most common symptoms, means of diagnosis and that it has increased prevalence among the poor. The results of the focus group discussions are summarised in box below.

Interviews with the DOTS workers : Most thought that there was adequate counselling for TB patients but thought that defaulters were still confused about their disease and treatment. They discussed that the reason why some patients consulted traditional healers was because the patients did not understand the inefficiency of traditional healers and another reason for consultation was because the patients valued the relationship with the traditional healer.

The respondents were of the opinion that there were no problems with the DOTS office timing as arrangements were made to keep it open after office hours. They thought that some of the main reasons for defaulting were social, stigma against the disease transportation and financial difficulties.

Interviews with patients : Most thought their knowledge about the disease and it treatment was inadequate and almost half said they had no counselling at all before being given treatment.

\section{Participant's opinions as to why patients defaulted from treatment}

- Alcoholics and smokers were not committed in taking caring of their own health, usually had a poor diet, often complained of weakness after treatment and may have had improper counselling and distribution of medicine.

- Patients may have felt better and so ceased medication

- Patients had difficulty walking long distances

- Patients having their own job had difficulty in going to the DOTS centers because of the fixed timing of the centers.

- There were improper storage systems and ineffective drugs at DOTS centres
- Private clinics were accessed for confidentiality reasons despite the lack of follow up

- A lack of knowledge in the community about TB, diet, medication and follow-up

- $\quad$ The use of traditional healers

- Younger patients and females in particular because of their fear of not being able to get married, may default because of the stigma associated with the disease

- Those from lower socio-economic groups who migrated to other areas or even other countries looking for work were less likely to complete treatment

- The elderly found it difficult to travel to the DOTS centre each day

- Lack of incentives for DOTS workers to do home visits and follow-up

Over half the patients were the family's main income earner and most defaulters cited insufficient income as a reason for not completing treatment. As compared to the opinion of the DOTS workers, only 12 patients thought they needed to hide their disease and most did not think that confidentiality was a reason for attending private clinics.

Distance from the DOTS clinic was more important factor than the DOTS office hours, despite the fact that all 35 of the defaulters worked from 10am - 5pm which were the only hours their local DOTS clinic remained open.

\section{DISCUSSION}

In the present study the defaulter rate in Sunsari district in 2002-03 was 2.5\% and the failure rate $0.7 \%$. The National Tuberculosis Control Programme, (NTP) Nepal aims for a defaulter rate of $<10 \%$ and a failure rate of $<4 \%{ }^{5}$ Other sources recommend much tighter control with rates of $<5 \%$ and $<1 \%$ respectively. ${ }^{5}$ The record of the NTP (1999-2000) for Sunsari District had default rate of $9 \%$ and failure rate of $2 \%{ }^{2}$ 
Despite excellent figures for Dharan and Sunsari district, TB is still a major Public Health issue for Nepal and much still remains to be done to have better control. As Médecins Sans Frontiers points out, not only is there much to be done with current tools, but new and better diagnostic tools and medications are urgently required worldwide. ${ }^{3}$ With the emergence of MDR-TB there is need to give increased importance for the completion of treatment.

All of the defaulter patients in this study were financially poor had trouble leaving work to go to the DOTS centre, as well as difficulty with distance and transport. Many were the families' chief income earner, all were in debt and a majority of the rural interviewees in this study thought distance from the clinic was a major problem. The problems associated with directly observed therapy in rural districts of Nepal were addressed recently by an article in the Lancet. The study found that family-member and community DOTS strategies had similar success rates to the usual directly observed therapy by DOTS workers. ${ }^{13}$

Many DOTS worker felt that lack of knowledge was one of the reasons for defaulting and many patients also felt that their knowledge on TB was below average. On the other hand most DOTS workers thought their counselling was adequate and the focus groups discussion revealed that the knowledge of the participants was reasonably accurate. The previous study in eastern Nepal had discussed about lack of knowledge on side effects of anti-TB drugs and also about the consequences of stopping TB treatment. ${ }^{8}$ General knowledge about TB was reasonable in both adherent and non-adherent groups but there is room for more education of both patients and the general community. ${ }^{13}$ In the DOTS centre from which there were no defaulters, all the workers were local people. Having local staff at the DOTS centres may improve the capacity of the center in giving health education and thus reduce the defaulters.

In one south Indian study $25 \%$ of patients almost two third of the defaulters were alcoholic. ${ }^{11}$ In our study the focus groups heavily emphasized alcohol as a major issue for defaulting treatment. Nearly half of the defaulters and treatment failures were spending money on alcohol.

Anumber of patients reported intermittent use of private clinics for privacy and also to avoid social stigma while visiting DOTS centre. Private clinics generally lack record keeping and follow up arrangements, and do not link records with those of the DOTS centre. Integrating TB care between all providers is another of the priority areas for the WHO 2006 "Stop TB" strategy. ${ }^{1}$

Home visiting may be a powerful tool to reduce the defaulter rate. After the researcher visited the homes of the 12 defaulters under one particular DOTS centre, 6 of them returned to treatment. A study in the Morang district of Nepal also reported that home visiting was very effective in retrieving patients. ${ }^{8}$

\section{CONCLUSION}

Based on the findings of this study, it is recommended that accessibility to quality services and improvement in patient education needs to be done. More DOTS centres with more flexible working hours are needed so that they are easily accessible to all patients. Adequate education to community and family-members on DOTS strategies is recommended. DOTS program should be introduced in all private clinics as a priority as suggested by the WHO. Home visits by the DOTS workers should be encouraged especially targeting working, elderly and severely ill patients.

TB will remain a major cause of morbidity and mortality in Nepal because of social issues like poverty, alcohol consumption, illiteracy, distance from health centres and stigma about the disease. These issues needs to be addressed appropriately and aggressively so that the country could contribute significantly in achieving the WHO goal of reducing the Global burden of TB. ${ }^{14}$ 


\section{ACKNOWLEDGEMENTS}

This research was done as part of Dr. Lamsal's Master of Family Medicine at B. P. Koirala Institute of Health Sciences, Dharan, Nepal. He wishes to thank all those who assisted him in this project.

\section{REFERENCES}

1. WHO, 2007. Global tuberculosis control - surveillance, planning, financing. Accessed January 2007 uww.who. inttb/en

2. National Tuberculosis Centre, 2000/01 Annual Report of Tuberculosis Control Programme Govt. of Nepal

3. Moran M. Medicines sans Frontiers, 2004 Campaign for access to Essential Medicines, Geneva.;(3):1-32

4. Kim HJ, Hong YP, 2001. Ambulatory treatment of multi -drug resistant pulmonary tuberculosis patients at a chest clinic. International Journal of Tuberculosis and Lung Disease;5(12): 1129-1136.

5. Editorial. Tuberculosis and Poverty, 2002. International Journal of Tuberculosis and Lung Disease; 6(9):745746.

6. Kumar M, Singh JV, 2002. Factors affecting the Non-Compliance in directly observed short course chemotherapy in Lucknow district. Indian Journal of Community Medicine; xxvii (3):114-117.

7. Buu TN, Lonnroth $K, 2003$. Initial defaulting in the National Tuberculosis Programme in Ho Chi Minh City Vietnam, a survey of extent reason and alternative actions taken following default. International Journal of Tuberculosis and Lung Disease;7(8):735-741.
8. Wares DF, Singh S, Acharya AK et al, 2003. Non adherence to tuberculosis treatment in the eastern Tarai of Nepal. International Journal of Tuberculosis and Lung Disease; 7(4):327-335

9. Molhotra R, Taneja DK, 2002. Awareness regarding tuberculosis in a rural population of Delhi. Indian Journal of Community Medicine.; xxvii(2): 62-68

10. Edginton ME, Sekatane CS, 2002. Patients' beliefs: do they affect tuberculosis control? A study in a rural district of South Africa. International Journal of Tuberculosis and Lung Disease;6(12):1075-1080.

11. Rajeswari R, Chandrasekaran V, 2002. Factors associated with patients and health system delay in the diagnosis of TB in South -India. International Journal of Tuberculosis and Lung Diseases;6(9):789795.

12. Rajeswari R, Balasubramanian R., 2002. Private Pharmacies in tuberculosis control -a neglected link. International Journal of Tuberculosis and Lung Disease;6(2):171-173

13. Bhal S, Mukharjee S, 1998. Unsupervised intermittent short course chemotherapy with intensive health education. Indian Journal of Tuberculosis;45(3):145-15

14. Newell JN, Baral SC, Pande SB, Bam DS, Malla P. 2006 Family-member DOTS and community DOTS for tuberculosis control in Nepal: cluster-randomised controlled trial. The Lancet Vol. 367, Issue 9514, pps 903-909 